\title{
Osteopontin expression in reactive lesions of gingiva
}

\author{
Rathinam ELANAGAI, Veeran VEERAVARMAL, Ramdas Madhavan NIRMAL
}

Division of Oral and Maxillofacial Pathology, Rajah Muthaiah Dental College and Hospital, Annamalai University, Annamalai Nagar, India.

Corresponding address: Veeran Veeravarmal - Division of Oral and Maxillofacial Pathology, Rajah Muthaiah Dental College and Hospital, Annamalai University - Annamalai Nagar - Tamil Nadu - India - Phone: 9443659412 - e-mail: varmal.opath@yahoo.com

Submitted: April 2, 2014 - Modification: September 28, 2014 - Accepted: September 30, 2014

\section{ABSTRACT}

\begin{abstract}
$\mathrm{R}$ eactive proliferations of the gingiva comprise lesions such as pyogenic granuloma (PG), inflammatory fibroepithelial hyperplasia (IFH), peripheral ossifying fibroma (POF), and peripheral giant cell lesion. Osteopontin (OPN) has a dual role, it promotes mineralization when it is bound to solid substrate, and on the other hand, it inhibits mineralization when it is seen in association with solution. Objectives: The study aimed to evaluate the expression of osteopontin in normal gingival tissue and different types of focal reactive proliferations of gingival tissue, and its role in the development of calcification within it. Material and Methods: The presence and distribution of osteopontin was assessed using immunohistochemistry in five cases of normal gingival tissue and 30 cases of focal reactive proliferations of gingiva. Results: There was no expression of osteopontin in normal subjects. Few cases of pyogenic granuloma, inflammatory fibroepithelial hyperplasia, and all the cases of peripheral ossifying fibroma showed positivity for osteopontin in the inflammatory cells, stromal cells, extracellular matrix, and in the calcifications. Conclusion: The expression of osteopontin in all the cases of peripheral ossifying fibroma speculates that the majority of the cases of peripheral ossifying fibroma originate from the periodontal ligament cells. The treatment modalities for peripheral ossifying fibroma should differ from other focal reactive proliferations of gingiva.
\end{abstract}

Keywords: Osteopontin. Immunohistochemistry.

\section{INTRODUCTION}

The oral mucosa is under constant barrage of irritation due to various external and internal stimuli culminating in a wide spectrum of developmental, inflammatory, reactive, and neoplastic diseases ${ }^{9}$. The reactive proliferations manifest both clinically and histologically as non-neoplastic nodular swellings. They occur as the aftermath of chronic and repeated tissue injury and present as an exuberant or excessive tissue response while regressing on elimination of the initiating factor ${ }^{9,21}$. Reactive lesions often present diagnostic challenges because of their overlapping and deceptive clinical presentation.

Reactive proliferations of the gingiva comprise lesions such as pyogenic granuloma (PG), inflammatory fibroepithelial hyperplasia (IFH), peripheral ossifying fibroma (POF), and peripheral giant cell lesion. Recently, localized juvenile spongiotic gingival hyperplasia has been added to this category ${ }^{4,20}$. These lesions develop in response to local irritants such as masticatory force, defective restorations, dental plaque, calculus, trauma, and iatrogenic factor. These irritational factors in conjunction with the peculiar anatomy of the gingiva attribute to the high incidence of reactive proliferation at this site. The most common reactive lesion is peripheral fibroma (56-61\%), followed in descending order by the pyogenic granuloma (19$27 \%)$, the peripheral ossifying fibroma $(10-18 \%)$, and peripheral giant cell lesion (1.5-7\%).

Long standing PG exhibits maturation and dystrophic calcifications that may mimic the histopathology of POF, which may be due to metaplastic changes observed in the fibroblasts ${ }^{18,22}$. Inflammatory fibroepithelial hyperplasia (IFH) is a hyperplastic fibrous tissue mass, usually located in the interdental papilla, and may be ascribed to 
irritation of periodontal ligament fibers. According to some investigators, it represents the immature variant of peripheral ossifying fibroma ${ }^{9}$.

Mineralization in POF can vary among cementumlike material, bone, and dystrophic calcification ${ }^{2,10,11}$. Whether all these reactive proliferations are separate entities or represent different stages in maturation of a single lesion has been a moot point for many years. Generally, the mineralization can be influenced by collagenous and non-collagenous proteins present in the extracellular matrix.

Osteopontin (OPN) is one such non-collagenous protein which is a highly phosphorylated sialoprotein and has high calcium binding potential. OPN is produced by osteoblasts at various stages of differentiation and by differentiated osteoblasts, osteocytes $^{15,23}$, and osteoclasts ${ }^{7}$. The OPN in the cemental lines may be utilized for osteoblasts cell adhesion or to promote early calcification events at this junction ${ }^{24}$. The OPN contains several serine phosphorylated sites and stretching of negatively charged aspartic acid residues responsible for the attraction of calcium, and also influence mineralization.

The persistence of focal reactive growth of gingiva for prolonged period may result in the formation of calcified structure within it. The initiating factor(s) influencing the dystrophic calcification or cementum or bone formation in these reactive lesions are poorly understood. The aim of the present study is to analyze the expression of osteopontin in normal gingival tissue and different types of focal reactive proliferations of gingiva in order to understand the possible role of osteopontin in the histogenesis of calcifications and ossifications in these lesions.

\section{MATERIAL AND METHODS}

This laboratory study consisted of 30 cases of histological variants of focal reactive proliferations that were subjected to histological examination in the Department of Oral and Maxillofacial Pathology, RMDC\&H, Annamalai University, Tamilnadu, India. These archival tissues and normal oral mucosa were fixed in $10 \%$ buffered formalin and embedded in paraffin wax, sections of $4 \mu \mathrm{m}$ thickness were prepared from each case for routine hematoxylin and eosin and immunohistochemical staining. Histopathological sections of the focal reactive proliferations were reviewed by the oral pathologists to confirm the diagnosis and to ascertain representative areas. Five cases of normal oral mucosa were deemed as a control group. All the 30 cases of focal reactive proliferations (10 cases each of PG, IFH, and POF) and the control group were subjected to immunohistochemistry to evaluate the expression of osteopontin.

Two to three serial sections of $3 \mu \mathrm{m}$ thickness were made and taken on APES pre-coated slides. The sections were deparaffinized and rehydrated in graded alcohol $(100 \%, 90 \%, 70 \%$, and $50 \%)$. The unmasking of antigen was done by the heat retrieval method in citrate buffer $(\mathrm{pH} \mathrm{6.5)}$. All the incubations were performed at room temperature using a humidifying chamber. At no time the tissue sections were allowed to dry during the staining procedure. After tapping off the excess buffer from the slides, the sections were sequentially treated with 3\% hydrogen peroxide (DAKO REAL En Vision, Glostrup, Denmark) and protein block (power block, DAKO REAL En Vision, Glostrup, Denmark) 10 minutes each, to suppress endogenous peroxidase activity and prevent unwanted protein binding. After thorough washing in PBS, the sections were incubated in Polyclonal rabbit anti-human osteopontin antibody (Thermoscientific, Marietta, Ohio, USA) for 30 minutes. The slides were washed and treated with Poly Horseradish peroxidase secondary antibody (DAKO REAL En Vision, Denmark) for 30 minutes. After thorough washing in PBS, the immunostaining was developed by treating with freshly constituted 3,3-diaminobenzidene tetra hydrochloride (DAB) solution for five minutes. After thorough washing in distilled water, the slides were counterstained with Mayer's hematoxylin and mounted with DPX.

The immunostained slides were evaluated by two blinded and calibrated pathologists independently. The positive areas were indicated by brown color precipitates and it was evaluated in stromal cells, extracellular matrix, calcifications, and inflammatory cells. All areas in each section were examined, and, in the areas where the intensity was predominant, these fields were taken into consideration. The intensity of staining (viewed at magnification of $10 x$ and $40 x$ ) was scored according to the following scale: 0 (no staining); 1 (mild staining); 2 (moderate staining); and 3 (intense staining). The data were analyzed using SPSS 21.0 software. The chi-square test was used to find the difference between the intensity levels and percentage positivity among the various groups. Differences with a probability value of $<0.05$ were considered statistically significant.

\section{RESULTS}

The histopathological findings of all the 30 cases of focal reactive proliferations are given in Figure 1. The five normal subjects did not show any area of inflammation, calcification, or ulceration. PG showed surface ulceration in five cases, and vascularity was a predominant feature in all the cases. In IFH, one case showed small globules of calcification in focal areas. The surface was not ulcerated in any of the cases of POF. All the 10 
cases exhibited calcifications which resembled trabecular bone in the majority of the cases, and few cases showed numerous basophilic cementumlike calcification. The collagen distribution is similar to IFH and showed equal number of cases with moderate to densely fibrous stroma.

\section{Osteopontin expression}

All the cases of normal oral mucosa showed no expression of OPN in the lamina propria. The expressions of OPN in all the 30 cases of focal reactive proliferations of gingiva are mentioned in the Figure 2. In $P G$, the stromal fibroblasts distributed along with inflammatory cells showed expression of OPN. Interestingly, the cells with cuboidal morphology and the matrix adjacent to them showed OPN expression in PG (Figure 3a). A focal collection of stromal cells, seen adjacent to blood vessel within the fibrovascular

\begin{tabular}{|c|c|c|c|c|c|c|c|c|c|c|c|c|}
\hline Histopathology diagnosis & $\begin{array}{l}\text { Total no. } \\
\text { of cases }\end{array}$ & \multicolumn{3}{|c|}{ Inflammation } & \multicolumn{3}{c|}{ Vascularity } & \multicolumn{3}{c|}{ Collagen } & \multicolumn{2}{c|}{ Calcification } \\
\hline & & Mild & Moderate & Intense & Mild & Moderate & Intense & Mild & Moderate & Intense & Present & Absent \\
\hline Pyogenic granuloma & 10 & - & 1 & 9 & - & - & 10 & 6 & 4 & - & - & 10 \\
\hline $\begin{array}{c}\text { Inflammatory fibroepithelial } \\
\text { hyperplasia }\end{array}$ & 10 & - & 4 & 6 & 3 & 3 & 4 & - & 5 & 5 & 1 & 9 \\
\hline Peripheral ossifying fibroma & 10 & - & 4 & 6 & 3 & 5 & 2 & - & 5 & 5 & 10 & - \\
\hline
\end{tabular}

Figure 1- Histopathological findings of pyogenic granuloma, inflammatory fibroepithelial hyperplasia, and peripheral ossifying fibroma

\begin{tabular}{|c|c|c|c|c|c|c|c|c|c|c|c|c|c|}
\hline $\begin{array}{c}\text { Histopathology } \\
\text { diagnosis }\end{array}$ & $\begin{array}{c}\text { Total no. } \\
\text { of cases }\end{array}$ & \multicolumn{3}{|c|}{ Inflammatory cells } & \multicolumn{4}{c|}{ Stromal cells } & \multicolumn{3}{c|}{$\begin{array}{c}\text { Extracellular matrix of } \\
\text { stromal tissue }\end{array}$} & \multicolumn{3}{c|}{ Calcification } \\
\hline & & Mild & Moderate & Intense & Mild & Moderate & Intense & Mild & Moderate & Intense & Mild & Moderate & Intense \\
\hline Normal oral mucosa & 5 & - & - & - & - & - & - & - & - & - & - & - & - \\
\hline Pyogenic granuloma & 10 & 7 & 1 & - & 3 & - & - & 3 & - & - & - & - & - \\
\hline $\begin{array}{c}\text { Inflammatory } \\
\text { fibroepithelial hyperplasia }\end{array}$ & 10 & 10 & - & - & 1 & - & - & - & - & - & 1 & - & - \\
\hline $\begin{array}{c}\text { Peripheral ossifying } \\
\text { fibroma }\end{array}$ & 10 & 10 & - & - & 10 & - & - & 10 & - & - & - & - & 10 \\
\hline
\end{tabular}

Figure 2- Osteopontin expression in pyogenic granuloma, inflammatory fibroepithelial hyperplasia, and peripheral ossifying fibroma

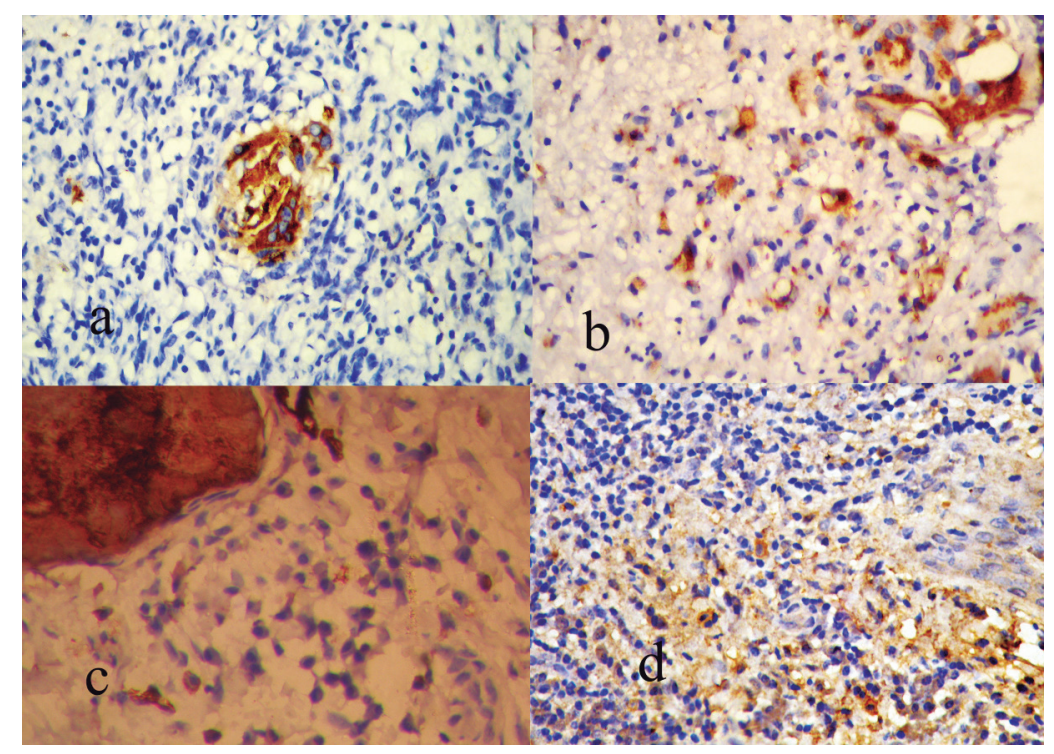

Figure 3- a) Pyogenic granuloma showing osteopontin (OPN) expression in stromal cells and extracellular matrix (40x); b) Higher magnification showing OPN expression in extracellular matrix of pyogenic granuloma; c) Inflammatory fibroepithelial hyperplasia (IFH) showing OPN expression in calcified structures, inflammatory and stromal cells (40x); d) OPN expression in inflammatory cells and in extracellular matrix of pyogenic granuloma (40x) 
component of PG, showed OPN expression. In PG, the extracellular matrix expression of OPN was observed in areas of inflammatory infiltrate. Lymphocytes, mast cells, macrophages, plasma cells, and osteoclastlike giant cells also exhibited positivity (Figure $3 d$ ). One case showed an area with osteoid appearance surrounded by stellate and cuboidal cells with few inflammatory cells that showed OPN expression in the cells with cuboidal and stellate morphology (Figure 3b).

The IFH showed OPN expression in the inflammatory component, stromal cells, and also in the calcification. None of the cases showed positivity in the extracellular matrix. The inflammatory cells such as lymphocytes, plasma cells, macrophages, and mast cells showed mild expression for OPN in focal areas in all cases of IFH. The calcification resembling dystrophic calcification was observed in close relation to chronic inflammatory cells in one area in IFH (Figure 3c).

The POF demonstrated OPN expression in the stromal cells with cuboidal or stellate morphology which were seen close to the calcified structures (Figure 4c). In all the cases of POF, the expression of OPN was observed in calcifications which resembled bone, cementum, and/or dystrophic calcification (Figures $4 a, b)$. All the cases of POF exhibited OPN expression in the extracellular matrix and the distribution was focal in nature (Figure 4d). The extracellular matrix positivity was observed predominantly in areas of inflammation and in areas of early bone formation (Figure 4d). Histopathologically, these later areas appeared as eosinophilic, extracellular matrix with less cellularity. There was focal and mild expression of OPN in the inflammatory cells of all the cases.

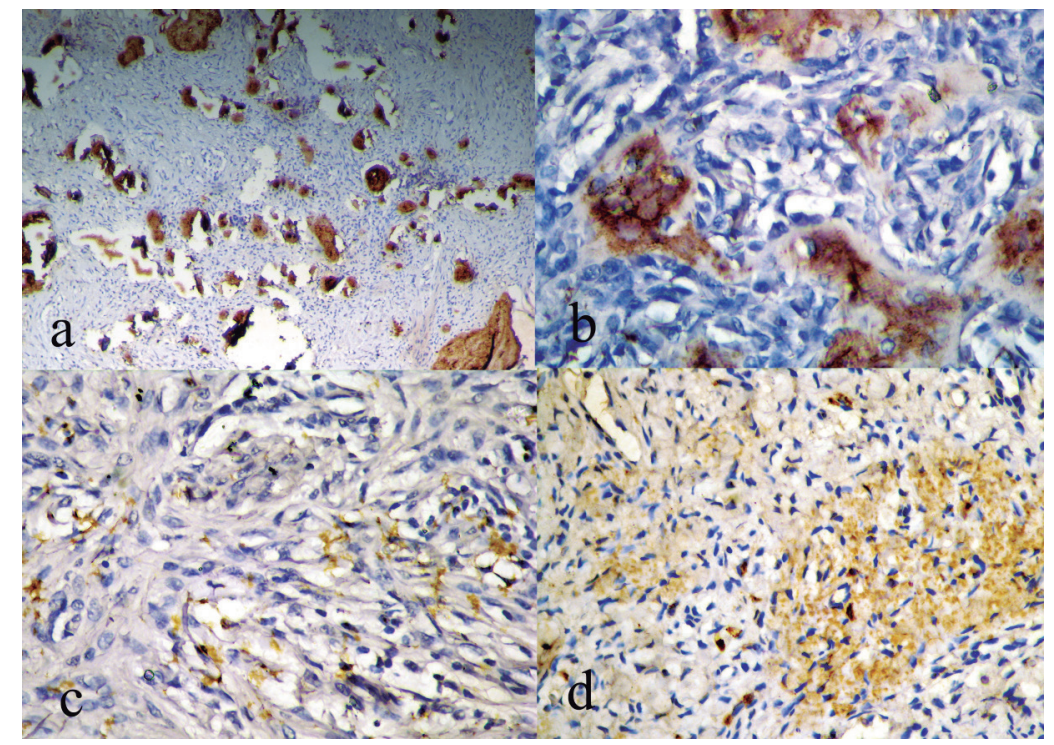

Figure 4- a) Peripheral ossifying fibroma (POF) showing osteopontin (OPN) expression in calcification resembling bone and cementum under lower magnification; b) The bony trabeculae expressing OPN in 40x; c) POF showing OPN expression in stromal cells (40x); d) The extracellular matrix of POF showing OPN expression under 40x

Table 1- Comparison of osteopontin expression between the control group and the study group using Chi-square test

\begin{tabular}{ccccc}
\hline Groups & Inflammatory cells & Stromal cells & Extracellular matrix & Calcification \\
\hline $\begin{array}{c}\text { Normal mucosa Vs. } \\
\text { PG }\end{array}$ & 0.014 & 0.171 & 0.171 & NIL \\
$\begin{array}{c}\text { Normal mucosa Vs. } \\
\text { POF }\end{array}$ & 0 & 0 & 0 & NIL \\
$\begin{array}{c}\text { Normal mucosa Vs. } \\
\text { IFH }\end{array}$ & 0 & No difference & 0.464 & NIL \\
PG Vs. POF & 0.171 & & 0.003 & NIL \\
PG Vs. IFH & 0.171 & 0.582 & 0.211 & NIL \\
IFH Vs. POF & No difference among \\
the group & 0 & 0 & 0 \\
\hline
\end{tabular}

PG=pyogenic granuloma; POF=peripheral ossifying fibroma; IFH=inflammatory fibroepithelial hyperplasia 
The comparison of OPN expression in the inflammatory component among normal and the PG, IFH, and POF was found to be statistically significant (Table 1). The statistical analysis showed no difference in OPN expression in the inflammatory infiltrate seen in IFH and POF. The expression of OPN was observed in all the cases of POF in the stromal tissue, while there was a total absence of expression in the fibroblast of the lamina propria of normal tissue. The statistical comparison between normal tissue and POF was found to be significant $(p<0.05)$ (Table 1$)$. The expression of OPN in the stromal cells is compared between PG and POF, on statistical evaluation the results were found to be significant $(p=0.0003)$. There was a marked difference in the expression of stromal cells for the OPN marker between IFH and POF $(p<0.05)$ (Table $1)$. There was no significance on comparing the expression of OPN in the stromal tissue of control tissue, $\mathrm{PG}$, and IFH.

The expression of OPN in the extracellular matrix of normal oral mucosa, PG, IFH showed no significance. On comparing the results of extracellular matrix expression of OPN in normal tissue and POF, it was observed to be highly significant $(p<0.05)$. The extracellular matrix of POF exhibited positive expression for OPN antibody in all the cases. Thus, the statistical comparison of the expression was found to be significant at $p$ value $(p=0.003)$. POF demonstrated expression in the extracellular matrix, on comparing the expression between IFH and POF; results were shown to be highly significant $(p<0.05)$ (Table 1). Histopathologically, there was total absence of calcification in oral mucosal tissue and PG. Furthermore, the expression of OPN in calcified tissue, when compared between IFH and POF, showed highly significant results $(p<0.05)$.

\section{DISCUSSION}

In the present study, 10 cases of PG exhibited high vascularity and inflammatory component distributed in loose fibrous connective tissues which ascertain its immature nature (Figure 1 ). Microscopically, IFH showed abundant collagen fibers with few to many blood vessels. Therefore, this group is composed of lesions in various stages of progression. The calcification was observed in one case of IFH, and was confined to a single site (Figure $1)$. The morphology of this calcification was akin to the dystrophic calcification described in chronic inflammatory conditions or the calcifications due to degenerating and senescent cells ${ }^{5}$.

The histogenesis of POF remains controversial. According to one view, it is believed to arise from the periodontal ligament tissue/periostium due to its increased occurrence in the gingiva, a tissue which is in proximity to the periodontal ligament ${ }^{18}$. Contrarily, it is also believed to be a mature PG and some features are attributed to the secondary changes due to repeated trauma ${ }^{1,18,20}$.

POF demonstrated fibrous proliferation with plenty of fibroblasts in association with mineralized tissue in the form of bone, cementum-like material, dystrophic calcification, or a combination of all ${ }^{20}$. Moderate to intense inflammatory cells were noted in most of the cases and vascularity was a predominant feature in cases which exhibited intense inflammation. Further it was observed that dense, mature collagen was seen only in cases with minimum vascularity. All cases showed calcification and fibrous connective tissue in equal proportion. The calcified structures resembled bony trabeculae with osteoid material in the periphery. Some mineralized structures presented as small globules mimicking dystrophic calcification or cementum (Figure 1).

The soft tissue possesses certain inhibitory factors/signals that prevent it from undergoing calcification. During disease process, there may be a deviation from the normal process or elimination of inhibitory factors leading to calcification of soft tissue ${ }^{5}$. In the present study, the lamina propria of the normal subjects showed no expression of OPN. When the normal tissue undergoes pathological changes, the OPN may be expressed in the stromal tissue ${ }^{14}$. This view is supported by the results obtained from the current study. OPN is expressed in activated chronic inflammatory cells such as lymphocytes, mast cells, and macrophages $3,6,13,17$. The PG, IFH, and POF expressed OPN in inflammatory infiltrate which included lymphocytes, macrophages, plasma cells, and mast cells morphology. The results of OPN expression in the inflammatory component between normal oral mucosa, PG, IFH, POF was found significant. This could be attributed to the reduction in the number of inflammatory component in normal oral mucosa.

Few giant cells seen in fibrous background of PG also showed OPN expression 7,15,23,28. Two possible explanations could be drawn for stromal cell positivity for OPN in PG. The first mechanism is that macrophages in the chronically inflamed tissue release pro-osteogenic cytokine, which stimulates the vascular smooth muscle cells to undergo osteogenic differentiation (or) release of calcifying matrix vesicle and initiate calcification ${ }^{26}$; the second mechanism may represent the immature POF without calcification. Thereby, this study speculates, based on the observations above, that the OPN expressing stromal cells may be osteoblast cells derived from PDL.

Nevertheless, it is not possible to state clearly whether it is inflammation induced osteogenesis 
or osteoblastic differentiation pertaining to PDL origin as a result of consistent association of these lesions with inflammation. Expression of OPN in extracellular matrix was observed in 3 cases of PG and all the cases of POF, whereas IFH failed to show any expression. Presumably, such areas could be the site of initiation of mineralization in the future. In one case of PG, OPN expression was confined to the extracellular matrix near the OPN positive stromal cells. In the other two cases, extracellular matrix positivity was seen in areas admixed with fibroblast and inflammatory cells. However, absence of OPN expression in the stromal cells in these two cases could be due to the release of OPN into the extracellular matrix. In POF, the areas of osteoid and the areas where the cells exhibited stellate morphology and separated apart, representing the initial phase of osteoid formation expressed OPN. These were the sites where the calcifications are initiated.

One case of IFH and all the 10 cases of POF showed OPN expression in the calcified structures resembling bone, cementum, and dystrophic calcification. According to Giachelli12 (1999), dystrophic calcification possesses several features of bone mineralization, including the presence of non-collagenous proteins such as OPN matrix GLA protein, Osteocalcin, SPARAC, and bone morphogenic protein. The data from the present study for OPN expression in calcified structure which resembled dystrophic calcification validates the view that the non-collagenous proteins have a role in mineralization ${ }^{8,12}$.

All the cases of POF showed expression of OPN in the calcifications resembling bone and/ or cementum. There was a significant difference in OPN expression in the mineralized component between POF and IFH. The mineralization seen in IFH was closely associated with inflammatory component. The inflammation induced dystrophic calcification is purportedly the mechanism involved in the formation of calcified structure in this group. The IFH having exhibited dystrophic ossification in one area may presumably represent maturing PG that passes through the stages of fibrous epulis and matures into fibroepithelial polyp ${ }^{27}$.

The information available in the literature and the results obtained from the present study insinuate that the calcified structures in POF resembled bone, cementum, and/or dystrophic calcification. The reason for the presence of calcified structures with morphology of bone and cementum in POF may be its tissue of origin. Alternatively, either fibroblastic metaplasia occurring in longstanding PG (or) osteogenic differentiation of smooth muscle cells are influenced by underlying inflammatory mechanism ${ }^{19,25}$. The combination of dystrophic calcification, bone and cementum in POF may be due to the occurrence of both the mechanisms simultaneously.

Except for a few cases, most of the cases of PG showed no expression of OPN in stromal cells and extracellular matrix, which contradicts the concept that the PG on maturation develops into POF. However, all the cases of POF subjected for OPN expression exhibited positivity in stromal cells, extracellular matrix, and calcification. From the results, we opine that the majority of POF are probably de novo in origin. The findings of the present study with regard to OPN expression in POF contradicted the results of Ono, et al. ${ }^{16}$ (2008), who observed OPN expression only in $50 \%$ of the cases of POF. The stromal cells exhibited stellate and/or cuboidal morphology, and cells in the periphery of ossification showed OPN expression, while such finding was not reported ${ }^{16}$.

The hypothesis given by many investigators for the varied histopathological presentation of focal reactive lesions implies different stages in maturation of a single entity. The results from the current study are in part concurrent with this concept. The epithelium present both in normal oral mucosa and in focal reactive proliferation is totally negative for OPN expression. The odontogenic cyst and tumors showed expression of OPN in the epithelium. The epithelium in reactive proliferation is not altered genetically, and that could be the reason for its absence.

After due cogitation, we reckon that the present study observed OPN expression in the stromal cells of PG, IFH, and POF. The results suggest that there is an osteoblastic differentiation of stromal cells in focal reactive proliferations of gingiva. The pyogenic granuloma showed OPN expression in the stromal cells and extracellular matrix in few cases, which favors the concept that pyogenic granuloma may mature into POF. However, POF showed OPN expression in stromal cells, extracellular matrix, and in areas of ossification in all the cases. This finding leads us to conclude that there is a definite veracity to the idea of co-definite osteoblast/ cementoblast differentiation and OPN plays a role in the development of ossification. The current study also theorizes that the majority of POF arises from the periodontal ligament cells. Hence, the treatment modalities for POF should differ from other focal reactive lesions of gingiva.

The data collected from the current study also upholds the view that PG may mature into IFH and POF on certain occasions. To ponder on the issue whether these lesions are separate entities or different phases during maturation of single entity, studies need to be carried out using specific markers involved in the differentiation of osteoblast, cementoblast, and in the development of ossification. 


\section{ACKNOWLEDGEMENT}

We would like to thank Dr. V. Srinivas Prasad, Post-graduate student in the Division of Oral and Maxillofacial Pathology, Rajah Muthaih Dental College and Hospital, Annamalai University, Annamalai Nagar, for language editing of the manuscript.

\section{REFERENCES}

1- Bhaskar SN, Jacoway JR. Peripheral fibroma and peripheral fibroma with calcification: report of 376 cases. J Am Dent Assoc. $1966 ; 73(6): 1312-20$

2- Buchner A, Hansen LS. The histomorphologic spectrum of peripheral ossifying fibroma. Oral Surg Oral Med Oral Pathol. 1987;63(4):452-61.

3- Cantor $\mathrm{H}$. The role of Eta-1/osteopontin in the pathogenesis of immunological disorders. Ann N Y Acad Sci. 1995;760:143-50.

4- Chang JY, Kessler HP, Wright JM. Localized juvenile spongiotic gingival hyperplasia. Oral Surg Oral Med Oral Pathol Oral Radiol Endod. 2008;106(3):411-8.

5- Cotran RS, Kumar V, Collins T, Robbins SL. Robbins \& Cotran pathologic basis of disease. $6^{\text {th }}$ ed. London: Saunders; 1999. $1425 \mathrm{p}$.

6- Denhardt DT, Mistretta D, Chambers AF, Krishna S, Porter JF, Raghuram S, et al. Transcriptional regulation of osteopontin and the metastatic phenotype: evidence for a Ras-activated enhancer in the human OPN promoter. Clin Exp Metastasis. 2003;20(1):7784.

7- Dodds RA, Connor JR, James IE, Rykaczewski EL, Appelbaum $E$, Dul E, et al. Human osteoclasts, not osteoblasts, deposit osteopontin onto resorption surfaces: an in vitro and ex vivo study of remodeling bone. J Bone Miner Res. 1995;10(11):1666-80. 8- Donley GE, Fitzpatrick LA. Noncollagenous matrix proteins controlling mineralization; possible role in pathologic calcification of vascular tissue. Trends Cardiovasc Med. 1998;8(5):199-206. 9- Effiom OA, Adeyemo WL, Soyele OO. Focal reactive lesions of the gingiva: an analysis of 314 cases at a tertiary health institution in Nigeria. Niger Med J. 2011;52(1):35-40.

10- Farquhar T, Maclellan J, Dyment $H$, Anderson RD. Peripheral ossifying fibroma: a case report. J Can Dent Assoc. 2008;74(9):80912.

11- García de Marcos JA, García de Marcos MJ, Arroyo Rodríguez S, Chiarri Rodrigo J, Poblet E. Peripheral ossifying fibroma: a clinical and immunohistochemical study of four cases. J Oral Sci. 2010;52(1):95-9.

12- Giachelli CM. Ectopic calcification: gathering hard facts about soft tissue mineralization. Am J Pathol. 1999;154(3):671-5.

13- Giachelli CM, Liaw L, Murry CE, Schwartz SM, Almeida M. Osteopontin expression in cardiovascular diseases. Ann N Y Acad Sci. 1995; 760:109-26.
14- Liu SJ, Hu GF, Liu YJ, Liu SG, Gao H, Zhang CS, et al. Effect of human osteopontin on proliferation, transmigration and expression of MMP-2 and MMP-9 in osteosarcoma cells. Chin Med J (Engl). 2004;117(2):235-40.

15- McKee MD, Nanci A. Postembedding colloidal-gold immunocytochemistry of noncollagenous extracellular matrix proteins in mineralized tissues. Microsc Res Tech. 1995;31(1):4462.

16- Ono N, Nakashima K, Rittling SR, Schipani E, Hayata T, Soma K, et al. Osteopontin negatively regulates parathyroid hormone receptor signaling in osteoblasts. J Biol Chem. 2008;283(28):19400-9.

17- Patarca R, Freeman GJ, Singh RP, Wei FY, Durfee T, Blattner F, et al. Structural and functional studies of the early $T$ lymphocyte activation 1 (Eta-1) gene. Definition of a novel T cell-dependent response associated with genetic resistance to bacterial infection. J Exp Med. 1989;170(1):145-61.

18- Patil K, Mahima VG, Lahari K. Extragingival pyogenic granuloma. Indian J Dent Res. 2006;17(4):199-202.

19- Peacock JD, Levay AK, Gillaspie DB, Tao G, Lincoln J. Reduced sox9 function promotes heart valve calcification phenotypes in vivo. Circ Res. 2010;106(4):712-9.

20- Rossmann JA. Reactive lesions of the gingiva: diagnosis and treatment options. Open Pathol J. 2011;5:23-32.

21- Shenoy SS, Dinkar AD. Pyogenic granuloma associated with bone loss in an eight year old child: a case report. J Indian Soc Pedod Prev Dent. 2006;24(4):201-3.

22- Shetty DC, Urs AB, Ahuja P, Hai HC, Sikka S, Sahu A, et al. A polarizing microscopic analysis of the calcified masses based on their collagen fibre orientation in peripheral ossifying fibroma. Pathol Lab Med Int. 2010;2:79-84.

23- Sodek J, Chen J, Nagata T, Kasugai S, Todescan R Jr, Li IW, et al. Regulation of osteopontin expression in osteoblasts. Ann N Y Acad Sci. 1995; 760:223-41.

24- Standal T, Borset M, Sundan A. Role of osteopontin in adhesion, migration, cell survival and bone remodeling. Exp Oncol. 2004;26(3):179-84

25- Steitz SA, Speer MY, Curinga G, Yang HY, Haynes P, Aebersold $R$, et al. Smooth muscle cell phenotypic transition associated with calcification: upregulation of $\mathrm{Cbfa} 1$ and downregulation of smooth muscle lineage markers. Circ Res. 2001;89(12):1147-54.

26- Ten Cate AR. Oral histology: development, structure, and function. $4^{\text {th }}$ ed. St. Louis: Mosby; 1994. 532 p.

27- Zain RB, Fei YJ. Fibrous lesions of the gingiva: a histopathologic analysis of 204 cases. Oral Surg Oral Med Oral Pathol. $1990 ; 70(4): 466-70$.

28- Zohar R, Lee W, Arora P, Cheifetz S, McCulloch C, Sodek J. Single cell analysis of intracellular osteopontin in osteogenic cultures of fetal rat calvarial cells. J Cell Physiol. 1997;170(1):88100 . 